01

\title{
Статистическое моделирование энергетических спектров электронов полевой эмиссии
}

\author{
(C) Н.В. Егоров, ${ }^{1}$ А.Ю. Антонов, ${ }^{2}$ Н.С. Демченко ${ }^{1}$ \\ ${ }^{1}$ Санкт-Петербургский государственный университет, \\ 199034 Санкт-Петербург, Россия \\ ${ }^{2}$ Санкт-Петербургский государственный электротехнический университет „ЛЭТИ“, \\ 197376 Санкт-Петербург, Россия \\ e-mail: robby7@mail.ru
}

(Поступило в Редакцию 30 марта 2016 г.)

Рассмотрен процесс моделирования случайных энергий электронов, покинувших источник в процессе полевой электронной эмиссии, на основе их энергетических спектров. Установлена связь между случайными значениями полной энергии и энергии, связанной с нормальной по отношению к поверхности компонентой импульса. Проанализированы семейства квадратурных формул, необходимые для интегрирования плотностей распределений частиц. Проведена статистическая проверка гипотезы о соответствии выборочных значений случайных энергий требуемым законам распределения.

DOI: 10.21883/JTF.2017.02.44122.1826

\section{Введение}

Источники электронов, работающие в режиме полевой эмиссии, находят все более широкое применение в различных областях науки и техники. Они обладают целым рядом достоинств, позволяющих выделить их на фоне термо- или фотокатодов [1]. Следует отметить прежде всего узость энергетического спектра (квазимонохроматичность) и ничтожное время отклика на изменение внешних управляющих параметров (значений потенциалов на вспомогательных электродах и аноде [2]). С другой стороны, полевые катоды очень чувствительны к состоянию поверхности, что накладывает требования на условия их работы - высокий уровень вакуума. Именно поэтому продолжаются поиски новых перспективных материалов для их использования [3].

Помимо анализа вольт-амперных характеристик важнейшей характеристикой полевого источника является спектр электронов [1,3]. Теория полевой электронной эмиссии Фаулера-Нордгейма, построенная для металлов, опиралась на распределение плотности потока частиц по энергиям, связанным с нормальной по отношению к поверхности составляющей импульса [4]. Понятно, что успешность сравнения теории и эксперимента в плане энергетических спектров зависит от конструкции энергоанализатора. В работе [5] теория полевой электронной эмиссии использует распределение потока частиц по полным энергиям. Связь этих двух спектров позволяет судить о начальных скоростях эмитированных частиц и об их угловом разбросе. Данная информация является крайне важной для моделирования движения частиц [6] и формирования пространственного заряда в межэлектродном пространстве [7].

Целью настоящей работы является моделирование энергии электрона как случайной величины в процессе полевой электронной эмиссии.

\section{Теоретическая часть}

В качестве физической модели рассмотрена плоская металлическая поверхность полевого катода, с которой вылетают электроны. Ось $z$ декартовой системы координат направлена по внешней нормали к поверхности. Пусть $p_{x}, p_{y}$ и $p_{z}$ - компоненты импульса электрона.

Отсчет энергий ведется от энергии покоя электрона в металле. В этих условиях можно считать, что $\mathscr{E} \in[0,+\infty)$.

Концентрация электронов в металле определяется произведением плотности энергетических состояний на вероятность данное состояние занять [5]:

$$
n(\mathscr{E}) d \mathscr{E}=\frac{4 \pi(2 m)^{\frac{3}{2}}}{h^{3}} \frac{\sqrt{\mathscr{E}} d \mathscr{E}}{1+\exp \left[\frac{\mathscr{E}-\mathscr{E}_{F}}{k T}\right]},
$$

где $m$ - масса электрона, $h-$ постоянная Планка, $\mathscr{E}_{F}-$ энергия Ферми, $k$ - постоянная Больцмана, $T-$ абсолютная температура (температура электронного газа).

Количество электронов с полными энергиями из интервала $[\mathscr{E}, \mathscr{E}+d \mathscr{E}]$ и энергией, связанной с нормальной компонентой импульса, из интервала $\left[\mathscr{E}_{z}, \mathscr{E}_{z}+d \mathscr{E}_{z}\right]$, падающих на единицу площади катода изнутри в единицу времени, определяется формулой [5]

$$
\begin{gathered}
N(\mathscr{E}) d \mathscr{E}_{z} d \mathscr{E}=\frac{4 \pi m}{h^{3}} \frac{d \mathscr{E}_{z} d \mathscr{E}^{\circ}}{1+\exp \left[\frac{\mathscr{E}-\mathscr{E}_{F}}{k T}\right]}, \\
\mathscr{E}=\mathscr{E}_{z}+\frac{p_{x}^{2}}{2 m}+\frac{p_{y}^{2}}{2 m} .
\end{gathered}
$$

Интегрирование выражения (1) по $\mathscr{E}_{z}$ от 0 до $\mathscr{E}$ дает распределение плотности потока по полным энергиям

$$
p_{\text {int }}(\mathscr{E}) d \mathscr{E}=\frac{4 \pi m}{h^{3}} \frac{\mathscr{E} d \mathscr{E}}{1+\exp \left[\frac{\mathscr{E}-\mathscr{E}_{F}}{k T}\right]}
$$


Аналогично интегрирование формулы (1) по $\mathscr{E}$ от $\mathscr{E}_{z}$ до $+\infty$ дает распределение плотности потока по нормальным компонентам энергии

$$
\nu_{\text {int }}\left(\mathscr{E}_{z}\right) d \mathscr{E}_{z}=\frac{4 \pi m k T}{h^{3}} \ln \left(1+\exp \left[-\frac{\mathscr{E}_{z}-\mathscr{E}_{F}}{k T}\right]\right) d \mathscr{E}_{z}
$$

Указанные распределения описывают статистику электронов внутри катода. Чтобы получить информацию об эмитированных частицах, необходимо функцию $N(\mathscr{E})$ домножить на коэффициент прохождения потенциального барьера $D\left(\mathscr{E}_{z}\right)[8]$ и снова провести интегрирование. Таким образом, внешняя плотность потока из интервала полных энергий равна

$$
p_{\mathrm{ext}}(\mathscr{E}) d \mathscr{E}=N(\mathscr{E}) d \mathscr{E} \int_{0}^{\mathscr{E}} D\left(\mathscr{E}_{z}\right) d \mathscr{E}_{z}
$$

Распределение электронов в выходящем потоке по $\mathscr{E}_{z}$ получается произведением „функции поставки“ $v_{\text {int }}\left(\mathscr{E}_{Z}\right)$ на вероятность туннелирования $D\left(\mathscr{E}_{z}\right)$ :

$$
\begin{aligned}
\mathcal{v}_{\mathrm{ext}}\left(\mathscr{\mathscr { O }}_{z}\right) d \mathscr{E}_{z}= & \frac{4 \pi m k T}{h^{3}} D\left(\mathscr{E}_{z}\right) \\
& \times \ln \left(1+\exp \left[-\frac{\mathscr{E}_{z}-\mathscr{E}_{F}}{k T}\right]\right) d \mathscr{E}_{z}
\end{aligned}
$$

Если произвести домножение любого внешнего распределения на элементарный заряд и провести интегрирование по всевозможным значениям энергии, то получим суммарный заряд, вылетающий с единицы поверхности катода в единицу времени, т.е. плотность эмиссионного тока $j$ :

$$
\begin{aligned}
j & =e \iint_{\Omega} N(\mathscr{E}) D\left(\mathscr{E}_{z}\right) d \mathscr{E}_{z} d \mathscr{E} \\
& =e \int_{0}^{+\infty} p_{\mathrm{ext}}(\mathscr{E}) d \mathscr{E}=e \int_{0}^{+\infty} v_{\mathrm{ext}}\left(\mathscr{E}_{z}\right) d \mathscr{E}_{z} .
\end{aligned}
$$

Область интегрирования

$$
\Omega=\left\{\left(\mathscr{E}_{z}, \mathscr{E}\right): 0 \leqslant \mathscr{E}_{z} \leqslant \mathscr{E}<+\infty\right\}
$$

Вылет отдельного электрона в силу множества факторов, на него влияющих, можно рассматривать как случайный процесс. Энергии $\mathscr{E}_{z}^{*}$ и $\mathscr{E}^{*}$, с которыми он покинул поверхность катода, - случайные величины. Дальнейшая задача заключается в моделировании и получении выборки указанных случайных величин.

\section{Плотности и функции распределений вероятностей}

Очевидно, что случайные величины $\mathscr{E}_{z}^{*}$ и $\mathscr{E}^{*}$ оказываются зависимыми. Согласно закону совместных вероятностей, величину $N(\mathscr{E})$ можно рассматривать как совместную плотность вероятностей с точностью до нормировочного множителя. Для всех трех внешних распределений нормировочный множитель одинаков и равен $e / j$. Совместную плотность можно представить в виде произведения условных плотностей.

Пусть координаты случайной точки $\mathbf{q}=$ $=\left(\xi_{1}, \xi_{2}, \ldots, \xi_{n}\right)$ зависимы. Тогда совместная плотность вероятностей может быть представлена в виде произведения условных плотностей

$$
\begin{gathered}
f_{\mathbf{q}}\left(x_{1}, x_{2}, \ldots, x_{n}\right)=f_{1}\left(x_{1}\right) \\
\times f_{2}\left(x_{2} \mid x_{1}\right) \ldots f_{n}\left(x_{n} \mid x_{1}, x_{2}, \ldots, x_{n-1}\right), \\
f_{1}\left(x_{1}\right)=\int \ldots \int f_{\mathbf{q}}\left(x_{1}, x_{2}, \ldots, x_{n}\right) d x_{2} d x_{3} \ldots d x_{n}, \\
f_{2}\left(x_{2} \mid x_{1}\right)=\frac{1}{f_{1}\left(x_{1}\right)} \int \ldots \int f_{\mathbf{q}}\left(x_{1}, x_{2}, \ldots, x_{n}\right) \\
\quad \times d x_{3} d x_{4} \ldots d x_{n}, \\
\ldots \\
f_{n}\left(x_{n} \mid x_{1}, x_{2}, \ldots, x_{n-1}\right) \\
=\frac{f_{\mathbf{q}}\left(x_{1}, x_{2}, \ldots, x_{n}\right)}{f_{1}\left(x_{1}\right) f_{2}\left(x_{2} \mid x_{1}\right) \ldots f_{n-1}\left(x_{n-1} \mid x_{1}, x_{2}, \ldots, x_{n-2}\right)} .
\end{gathered}
$$

Условные функции распределения вводятся по правилу

$$
F_{i}\left(x_{i} \mid x_{1}, x_{2}, \ldots, x_{i-1}\right)=\int_{-\infty}^{x_{i}} f_{i}\left(y \mid x_{1}, x_{2}, \ldots, x_{i-1}\right) d y .
$$

Совокупность случайных чисел $\xi_{1}, \xi_{2}, \ldots, \xi_{n}$, полученных при последовательном решении уравнений

$$
\begin{aligned}
F_{1}\left(\xi_{1}\right)=\gamma_{1}, & F_{2}\left(\xi_{2} \mid \xi_{1}\right)=\gamma_{2}, \ldots, \\
& F_{n}\left(\xi_{n} \mid \xi_{1}, \xi_{2}, \ldots, \xi_{n-1}\right)=\gamma_{n},
\end{aligned}
$$

имеет совместную плотность вероятностей $f_{\mathbf{q}}\left(x_{1}, x_{2}, \ldots, x_{n}\right)$ [9]. Здесь $\gamma_{i}-$ независимые реализации случайной величины, равномерно распределенной на интервале $[0,1)$. Очевидно, что количество представлений совместной плотности через условные равно $n$ ! - число перестановок координат. Сложность получающейся системы уравнений зависит от выбора конкретного представления.

Используя такой подход к формуле (1), необходимо одновременно разыгрывать полную энергию $\mathscr{E} *$ и энергию $\mathscr{E}_{z}^{*}$, связанную с нормальной компонентой импульса. Возможны два варианта представления совместной плотности распределения. Встает вопрос - какую из координат выбрать в качестве ведущей? В настоящей работе рассмотрены оба случая.

Пусть $x_{1}=\mathscr{E}$, тогда плотность вероятностей $f_{1}(\mathscr{E})$ вычисляется следующим образом (важно не забывать об ограничениях, фигурирующих в области $\Omega$ ):

$$
f_{1}(\mathscr{E})=\widetilde{p}_{\text {ext }}(\mathscr{E})=A \int_{0}^{\mathscr{E}} N(\mathscr{E}) D\left(\mathscr{E}_{z}\right) d \mathscr{E}_{z}=A p_{\text {ext }}(\mathscr{E})
$$




$$
A=\frac{e}{j},
$$

где $\widetilde{p}_{\text {ext }}(\mathscr{E})-$ плотность вероятностей распределения эмитированных электронов по полным энергиям $\mathscr{E}$. Вторая (условная) плотность вероятностей имеет вид

$$
f_{2}\left(\mathscr{E}_{z} \mid \mathscr{E}\right)=\frac{A N(\mathscr{E}) D\left(\mathscr{E}_{z}\right)}{A p_{\mathrm{ext}}(\mathscr{E})}=D\left(\mathscr{E}_{z}\right)\left[\int_{0}^{\mathscr{E}} D\left(\mathscr{E}_{z}\right) d \mathscr{E}_{z}\right]^{-1}
$$

В этих условиях функции распределения для $f_{1}(\mathscr{E})$ и $f_{2}\left(\mathscr{E}_{z} \mid \mathscr{E}\right)$ представлены как (для переменной интегрирования и переменного предела будет использоваться одно обозначение)

$$
\begin{aligned}
F_{1}(\mathscr{E}) & =\int_{0}^{\mathscr{E}} f_{1}(\mathscr{E}) d \mathscr{E}=A \int_{0}^{\mathscr{E}} p_{\text {ext }}(\mathscr{E}) d \mathscr{E}, \\
F_{2}\left(\mathscr{E}_{z} \mid \mathscr{E}\right) & =\int_{0}^{\mathscr{E}_{z}} f_{2}\left(\mathscr{E}_{z} \mid \mathscr{E}^{\mathscr{E}}\right) d \mathscr{E}_{z} \\
& =\int_{0}^{\mathscr{E}_{z}} D\left(\mathscr{E}_{z}\right) d \mathscr{\mathscr { E }}_{z}\left[\int_{0}^{\mathscr{E}} D\left(\mathscr{\mathscr { E }}_{z}\right) d \mathscr{\mathscr { E }}_{z}\right]^{-1} .
\end{aligned}
$$

Метод обратных функций распределения дает уравнения для последовательного определения случайных значений энергий $\mathscr{E}^{* *}$ и $\mathscr{E}_{z}^{\mathscr{*}}$ :

$$
\int_{0}^{\mathscr{E}^{*}} \widetilde{p}_{\text {ext }}(\mathscr{E}) d \mathscr{E}=\gamma_{1}, \quad \int_{0}^{\mathscr{E}_{z}^{*}} D\left(\mathscr{E}_{z}\right) d \mathscr{E}_{z}=\gamma_{2} \int_{0}^{\mathscr{E}^{*}} D\left(\mathscr{E}_{z}\right) d \mathscr{E}_{z} .
$$

Оба уравнения необходимо решить численно относительно неизвестного верхнего предела, после чего можно получить случайные величины с требуемыми законами распределения.

Во втором случае, когда $x_{1}=\mathscr{E}_{z}$, процедура приведет к другим уравнениям. Плотность вероятностей $f_{1}\left(\mathscr{E}_{z}\right)$ вычисляется следующим образом:

$$
\begin{aligned}
f_{1}\left(\mathscr{E}_{z}\right) & =\widetilde{v}_{\text {ext }}\left(\mathscr{E}_{z}\right)=A \int_{\mathscr{E}_{z}}^{+\infty} N(\mathscr{E}) D\left(\mathscr{E}_{z}\right) d \mathscr{E} \\
& =A D\left(\mathscr{E}_{z}\right) v_{\text {int }}\left(\mathscr{E}_{z}\right)=A v_{\text {ext }}\left(\mathscr{E}_{z}\right),
\end{aligned}
$$

где $\widetilde{\mathcal{v}}_{\text {ext }}\left(\mathscr{E}_{z}\right)-$ плотность вероятностей распределения электронов по энергиям $\mathscr{E}_{z}$. Вторая (условная) плотность вероятностей имеет вид

$$
f_{2}\left(\mathscr{E} \mid \mathscr{E}_{z}\right)=\frac{A N(\mathscr{E}) D\left(\mathscr{E}_{z}\right)}{A v_{\text {ext }}\left(\mathscr{E}_{z}\right)}=\frac{N\left(\mathscr{E}^{\mathscr{E}}\right)}{v_{\text {int }}\left(\mathscr{E}_{z}\right)}
$$

Тогда функции распределения для $f_{1}\left(\mathscr{E}_{z}\right)$ и $f_{2}\left(\mathscr{E}_{\mid} \mid \mathscr{E}_{z}\right)$ будут представлены выражениями

$$
F_{1}\left(\mathscr{E}_{z}\right)=\int_{\mathscr{E}_{z}}^{+\infty} f_{1}\left(\mathscr{E}_{z}\right) d \mathscr{E}_{z}=A \int_{\mathscr{E}_{z}}^{+\infty} v_{\text {ext }}\left(\mathscr{E}_{z}\right) d \mathscr{E}_{z}
$$

$$
\begin{aligned}
F_{2}\left(\mathscr{E} \mid \mathscr{E}_{z}\right) & =\int_{\mathscr{E}}^{+\infty} f_{2}\left(\mathscr{\mathscr { E }} \mid \mathscr{E}_{z}\right) d \mathscr{E} \\
& =\frac{1}{v_{\text {int }}\left(\mathscr{E}_{z}\right)} \int_{\mathscr{E}}^{+\infty} N(\mathscr{E}) d \mathscr{E}=\frac{v_{\text {int }}(\mathscr{E})}{v_{\text {int }}\left(\mathscr{E}_{z}\right)}
\end{aligned}
$$

Далее осуществляется переход к уравнениям для последовательного определения случайных значений энергий $\mathscr{E}_{z}$ и $\mathscr{E}^{*}:$

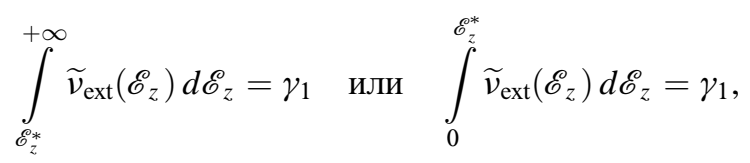

так как величины $\gamma$ и $(1-\gamma)$ распределены одинаково, и

$$
v_{\text {int }}\left(\mathscr{E}^{*}\right)=\gamma_{2} v_{\text {int }}\left(\mathscr{E}_{z}^{*}\right) .
$$

Решая уравнение (6) численно, можно получить слу-

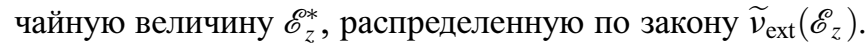
Уравнение (7) имеет аналитическое решение, поэтому очевидно, что второй подход более простой и экономичный в плане используемых вычислительных ресурсов. Следовательно, разумнее выбрать в качестве ведущей величины энергию $\mathscr{E}_{z}$.

Вышеизложенные результаты удобно представить в виде утверждения.

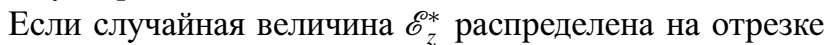
$[0,+\infty)$ с плотностью вероятностей $\widetilde{v}_{\text {ext }}\left(\mathscr{E}_{z}\right)$, то случайная величина

$$
\mathscr{E}^{*}=\mathscr{E}_{F}-k T \ln \left[\left(1+\exp \left[-\frac{\mathscr{E}_{z}^{*}-\mathscr{E}_{F}}{k T}\right]\right)^{\gamma}-1\right]
$$

распределена на том же отрезке с плотностью вероятностей $\widetilde{p}_{\text {ext }}(\mathscr{E})$, где $\gamma-$ равномерно распределенная на отрезке $[0,1)$ случайная величина.

Выражение (8) можно упростить в предельных случаяX

$$
\mathscr{E}^{\mathscr{*}}=\left\{\begin{array}{lll}
\mathscr{E}_{z}^{* *}-k T \ln \gamma, & \text { если } & \exp \left[-\frac{\mathscr{E}_{z}^{*}-\mathscr{E}_{F}}{k T}\right] \ll 1, \\
\gamma \mathscr{E}_{z}^{*}+(1-\gamma) \mathscr{E}_{F}, & \text { если } & \exp \left[-\frac{\mathscr{E}_{z}^{*}-\mathscr{E}_{F}}{k T}\right] \gg 1
\end{array}\right.
$$

\section{Методы интегрирования}

Согласно предлагаемой схеме моделирования случайных энергий с законами распределения $\widetilde{v}_{\text {ext }}\left(\mathscr{E}_{z}\right)$ и $\widetilde{p}_{\text {ext }}(\mathscr{E})$, требуется численно решить уравнение (6) относительно верхнего предела. Если использовать квадратурную формулу, то значения подынтегральной функции (а конкретно прозрачности барьера $\left.D\left(\mathscr{E}_{z}\right)\right)$ в заданных абсциссах можно получить, решая уравнение Шредингера в векторном виде. Схожая постановка задачи изложена в [8] и специально не будет рассматриваться в настоящей работе. Можно лишь отметить, что авторами был 
Таблица 1. Веса $w_{i}$ и коэффициенты $k$ для методов интегрирования

\begin{tabular}{l|c|c|c|c|c|c|c|c|c|c}
\hline \multicolumn{1}{c|}{ Метод } & $w_{1}$ & $w_{2}$ & $w_{3}$ & $w_{4}$ & $w_{5}$ & $w_{6}$ & $w_{7}$ & $\cdots$ & $w_{n}$ & $k$ \\
\hline Прямоугольников (r) & 0 & 1 & 0 & 1 & 0 & 1 & 0 & $\ldots$ & 0 & 2 \\
Трапеций (t) & 1 & 2 & 2 & 2 & 2 & 2 & 2 & $\cdots$ & 1 & $1 / 2$ \\
Симпсона (s) & 1 & 4 & 2 & 4 & 2 & 4 & 2 & $\ldots$ & 1 & $1 / 3$ \\
Боде (b) & 7 & 32 & 12 & 32 & 14 & 32 & 12 & $\ldots$ & 7 & $2 / 45$
\end{tabular}

Таблица 2. Вычисление $I(x)=\int_{0}^{x} \sin x d x ; x \in(0, \pi)$

\begin{tabular}{c|c|c|c|c|c|c}
\hline \multirow{2}{*}{ Метод } & \multicolumn{3}{|c|}{$t o l=10^{-6}$} & \multicolumn{3}{c}{ tol $=10^{-9}$} \\
\cline { 2 - 7 } & $n$ & $\varepsilon_{\max }$ & $\langle\varepsilon\rangle$ & $n$ & $\varepsilon_{\max }$ & $\langle\varepsilon\rangle$ \\
\hline $\mathrm{rsb}$ & 2049 & $1.03 \cdot 10^{-13}$ & $1.14 \cdot 10^{-14}$ & 65537 & $1.16 \cdot 10^{-12}$ & $6.39 \cdot 10^{-13}$ \\
$\mathrm{ttb}$ & 2049 & $1.03 \cdot 10^{-13}$ & $1.14 \cdot 10^{-14}$ & 65537 & $1.16 \cdot 10^{-12}$ & $6.39 \cdot 10^{-13}$ \\
$\mathrm{tsb}$ & 1025 & $1.23 \cdot 10^{-13}$ & $9.66 \cdot 10^{-15}$ & 32769 & $3.59 \cdot 10^{-13}$ & $1.60 \cdot 10^{-13}$ \\
$\mathrm{ssb}$ & 65 & $4.21 \cdot 10^{-7}$ & $6.74 \cdot 10^{-9}$ & 513 & $2.02 \cdot 10^{-12}$ & $3.09 \cdot 10^{-14}$ \\
$\mathrm{ss}$ & 33 & $2.25 \cdot 10^{-3}$ & $5.67 \cdot 10^{-5}$ & 257 & $1.29 \cdot 10^{-6}$ & $2.04 \cdot 10^{-8}$ \\
$\mathrm{bb}$ & 17 & $2.22 \cdot 10^{-4}$ & $9.77 \cdot 10^{-6}$ & 65 & $4.21 \cdot 10^{-8}$ & $6.74 \cdot 10^{-9}$
\end{tabular}

использован метод Рунге-Кутты-Нюстрема с коэффициентами Дорманда-Принса 12-го порядка [10]. Для интегрирования были использованы методы с равноотстоящими узлами, поскольку погрешность интерполяции, необходимой для отыскания верхнего предела, зависит от расстояния между абсциссами.

Классический подход к оценке погрешности дает правило Рунге, согласно которому вычисления следует производить с нормальным и удвоенным шагами. Каждый раз количество интервалов, на которое разбивается отрезок интегрирования, увеличивается в 2 раза. Вычисления проводятся до тех пор, пока не будет выполнено неравенство

$$
\frac{\left|I_{2}-I_{1}\right|}{2^{p}-1} \leqslant \varepsilon\left|I_{2}\right|
$$

что означает достижение требуемой точности. Здесь $I_{1}$ и $I_{2}$ - результаты вычислений с шагом $h$ и с шагом $2 h$ соответственно, а $p$ - порядок используемого метода интегрирования.

В качестве альтернативного метода оценки погрешности использовался известный подход, опирающийся на три оценки интеграла $I_{1}, I_{2}$ и $I_{3}$ разной степени точности $\left(I_{3}-\right.$ самый точный метод). Шаг $h$ также уменьшается вдвое до тех пор, пока не достигается требуемый уровень погрешности вычислений [11]

$$
\left|I_{1}-I_{2}\right| \leqslant \varepsilon\left|I_{3}\right| \text {. }
$$

В настоящей работе рассмотрены составные формулы прямоугольников, трапеций, Симпсона и Боде. Квадратурные формулы более высокой степени точности нами не рассматривались по причине наличия в них отрицательных весовых коэффициентов, что часто делает работу таких методов неустойчивой. Общая формула для четырех выбранных методов имеет следующий вид:

$$
\int_{a}^{b} f(x) d x=k h \sum_{i=1}^{n} w_{i} f\left(x_{i}\right), \quad h=\frac{b-a}{n-1} .
$$

Значения весов $w_{i}$ и коэффициента $k$ для составных формул представлены в табл. 1.

Для выбора метода оценки погрешности и методов, которые будут использоваться для решения поставленной задачи, проводилось интегрирование нескольких функций, интегралы от которых могут быть получены аналитически. Выбор функций диктовался характером формы энергетических распределений. Результаты для некоторых комбинаций методов и для двух различных заданных уровней погрешности вычисления (tol)

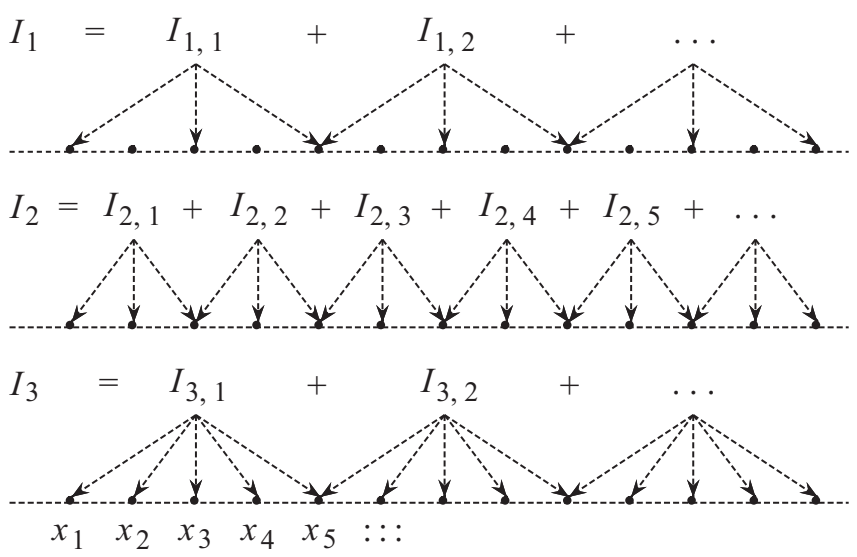

Рис. 1. Составные квадратурные формулы. 
Таблица 3. Вычисление $I(x)=\int_{-1}^{x} \frac{1}{1+100 x^{2}} d x ; x \in(-1,1)$

\begin{tabular}{c|c|c|c|c|c|c}
\hline \multirow{2}{*}{ Метод } & \multicolumn{3}{|c|}{$t o l=10^{-6}$} & \multicolumn{3}{c}{ tol $=10^{-9}$} \\
\cline { 2 - 7 } & $n$ & $\varepsilon_{\max }$ & $\langle\varepsilon\rangle$ & $n$ & $\varepsilon_{\max }$ & $\langle\varepsilon\rangle$ \\
\hline $\mathrm{rsb}$ & 513 & $1.89 \cdot 10^{-9}$ & $9.77 \cdot 10^{-11}$ & 16385 & $2.60 \cdot 10^{-14}$ & $1.72 \cdot 10^{-15}$ \\
$\mathrm{ttb}$ & 513 & $1.89 \cdot 10^{-9}$ & $9.77 \cdot 10^{-11}$ & 16385 & $2.60 \cdot 10^{-14}$ & $1.72 \cdot 10^{-15}$ \\
$\mathrm{tsb}$ & 257 & $2.21 \cdot 10^{-7}$ & $8.83 \cdot 10^{-9}$ & 8193 & $2.67 \cdot 10^{-14}$ & $2.04 \cdot 10^{-15}$ \\
$\mathrm{ssb}$ & 257 & $2.21 \cdot 10^{-7}$ & $8.83 \cdot 10^{-9}$ & 513 & $1.89 \cdot 10^{-9}$ & $9.77 \cdot 10^{-11}$ \\
$\mathrm{ss}$ & 257 & $2.68 \cdot 10^{-6}$ & $1.71 \cdot 10^{-7}$ & 257 & $2.68 \cdot 10^{-6}$ & $1.71 \cdot 10^{-7}$ \\
$\mathrm{bb}$ & 257 & $2.21 \cdot 10^{-7}$ & $8.83 \cdot 10^{-9}$ & 513 & $1.89 \cdot 10^{-9}$ & $9.77 \cdot 10^{-11}$
\end{tabular}

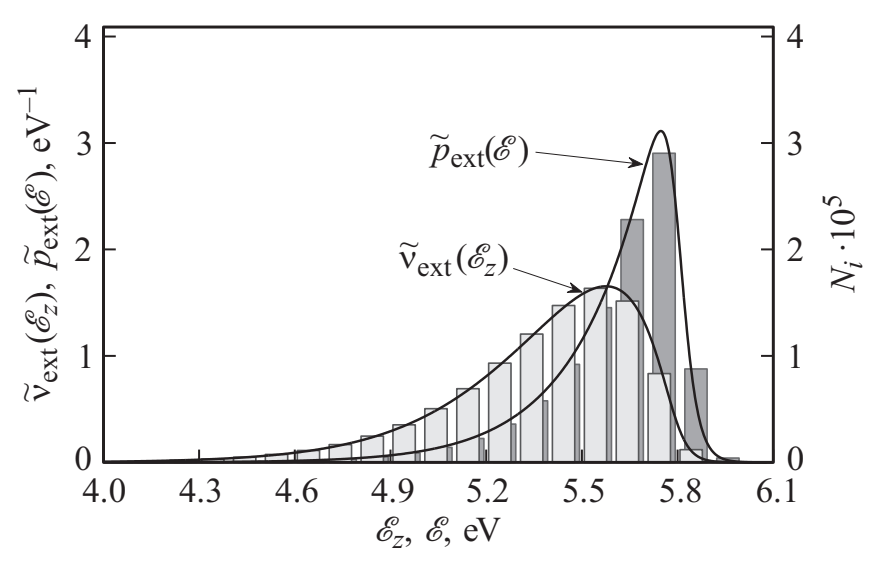

Рис. 2. Сравнение генеральной и выборочной плотностей распределения.

приведены в табл. 2 и 3. Число $n$ показывает количество интервалов, на которое оказался поделен отрезок интегрирования. Величина $\langle\varepsilon\rangle$ представляет собой среднее значение погрешности, вычисленное по ста точкам. Здесь важно отметить, что эти точки могли не совпадать с узлами (абсциссами) интегрирования, и в этом случае получались интегрированием интерполянта соответствующей степени (интерполянт четвертой степени для метода Боде, второй - для Симпсона).

Видно, что правило Рунге (двухбуквенное сочетание) для оценки погрешности годится не всегда. Подход, основанный на трех различных оценках интеграла, часто переоценивает погрешность (особенно при использовании грубых методов). За счет этого увеличивается количество интервалов и, следовательно, время счета. По этой причине выбор был остановлен на так называемой „золотой середине“ - комбинации методов Симпсон-Симпсон-Боде. С одной стороны, метод выдерживает заданный уровень точности, а с другой - не слишком мелко дробит заданный интервал.

Работа выбранной тройки методов проиллюстрирована на рис. 1. Здесь $I_{1}-$ оценка интеграла методом Симпсона с удвоенным шагом, $I_{2}-$ методом Симпсона с шагом $h, I_{3}$ - методом Боде.
Используемые квадратуры для 5-ти точек имеют вид:

$$
\begin{aligned}
& \int_{x_{1}}^{x_{5}} f(x) d x \approx I_{1,1}=\frac{2 h}{3}\left(f_{1}+4 f_{3}+f_{5}\right), \\
& \int_{x_{1}}^{x_{5}} f(x) d x \approx I_{2,1}+I_{2,2} \\
&=\frac{h}{3}\left(f_{1}+4 f_{2}+2 f_{3}+4 f_{4}+f_{5}\right), \\
& \int_{x_{1}}^{x_{5}} f(x) d x \approx I_{3,1} \\
&=\frac{2 h}{45}\left(7 f_{1}+32 f_{2}+12 f_{3}+32 f_{4}+7 f_{5}\right) .
\end{aligned}
$$

Количество узлов $n$ в составной формуле получается регламентированным. Для оценки интеграла по правилу Боде можно записать

$$
\int_{a}^{b} f(x) d x \approx I_{3}=I_{3,1}+I_{3,2}+\ldots+I_{3, M}, \quad n=4 M+1 .
$$

Промежуточные значения оценки интеграла могут быть получены аналитическим интегрированием интерполянта 4-й степени

$$
I(x)=\sum_{j=1}^{i-1} I_{3, j}+S_{i}(x), \quad i=4\left\lfloor\frac{x-a}{4 h}\right\rfloor+1 .
$$

Интегральный интерполяционный полином имеет вид

$$
\begin{aligned}
& S_{i}(x)= \\
& =t\left[f_{i}+t\left[\frac{1}{24}\left(-25 f_{i}+48 f_{i+1}-36 f_{i+2}+16 f_{i+3}-3 f_{i+4}\right)\right.\right. \\
& +t\left[\frac{1}{72}\left(35 f_{i}-104 f_{i+1}+114 f_{i+2}-56 f_{i+3}+11 f_{i+4}\right)\right. \\
& +t\left[\frac{1}{48}\left(-5 f_{i}+18 f_{i+1}-24 f_{i+2}+14 f_{i+3}+3 f_{i+4}\right)\right. \\
& \left.\left.\left.\left.+t\left[\frac{1}{120}\left(f_{i}-4 f_{i+1}+6 f_{i+2}-4 f_{i+3}+f_{i+4}\right)\right]\right]\right]\right]\right]
\end{aligned}
$$

где $t=\left(x-x_{i}\right) / h$. 
Для моделирования случайных значений $\mathscr{E}_{z}^{*}$ проводилось решение уравнения (6) методом дихотомии. Для моделирования случайных величин $\gamma$ был использован генератор псевдослучайных чисел Mersenne Twister (МТ19937) [12]. Далее по формуле (8) вычислялась реализация случайной величины $\mathscr{\mathscr { E }} *$. Были получены выборки разного объема $N$. Сравнение выборочной (гистограммы) и генеральной плотностей распределения приведено на рис. 2 для $N=10^{6}$ статистических испытаний. Видно, что полученные выборки хорошо соответствуют моделируемым законам распределения. Здесь напряженность электрического поля $E=5.0 \cdot 10^{9} \mathrm{~V} / \mathrm{m}$, температура $T=300 \mathrm{~K}$, работа выхода $\Phi=4.5 \mathrm{eV}$, энергия Ферми $\mathscr{E}_{\mathrm{F}}=5.78 \mathrm{eV}$ (последние два параметра соответствуют вольфраму).

\section{Статистическая проверка}

Визуальный анализ, представленный на рис. 2, не дает количественной оценки. Для ее получения и проверки гипотезы о соответствии выборки генеральной совокупности с функцией распределения был выбран критерий Колмогорова-Смирнова [13]. Критерий Пирсона сравнения плотностей распределения требует введения дополнительного параметра - числа интервалов для построения гистограммы. Критерий Крамера-фон Мизеса-Смирнова сложнее в реализации. Статистика критерия для конечной выборки рассчитывается по формуле

$$
D_{N}=\max _{1 \leqslant k \leqslant N}\left[\left|F_{k}-\frac{k-1}{N}\right|,\left|F_{k}-\frac{k}{N}\right|\right],
$$

где $F_{k}$ - значения функции распределения в точках вариационного ряда. В нашем случае $F_{k}$ вычислялись с помощью интегрального интерполянта по формулам

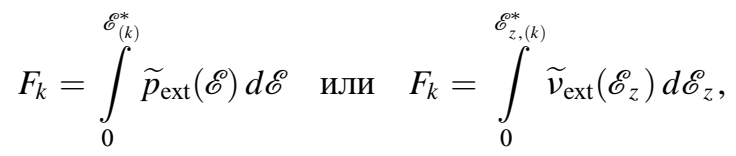

где выборочные значения $\mathscr{E}_{(k)}^{*}$ и $\mathscr{E}_{z,(k)}^{*}$ отсортированы по возрастанию. Сравнение генеральных и выборочных (ступенчатых) функций распределения для $N=100$ представлено на рис. 3. Видно, что даже при небольшом объеме выборки рассматриваемые функции не сильно отклоняются друг от друга.

Для принятия решения статистику критерия необходимо сравнить с величиной $D_{N}(\alpha)$, где $\alpha$ - заданный

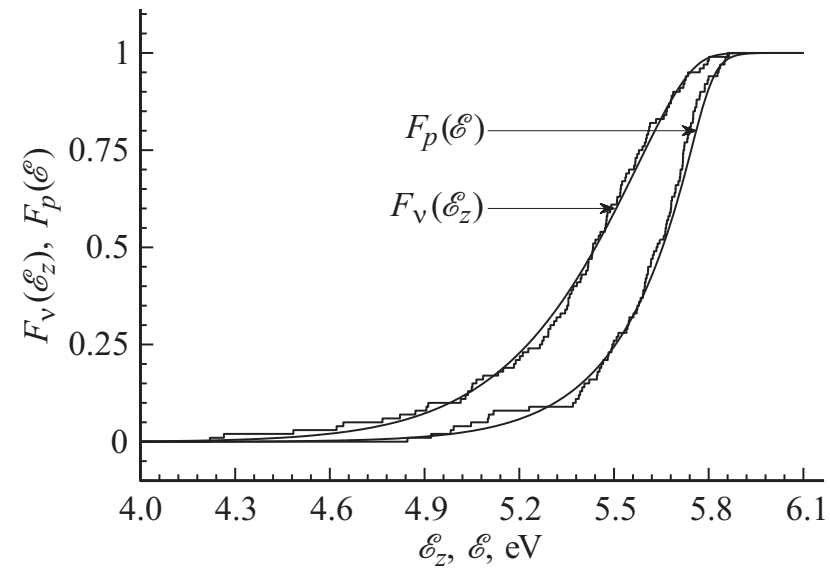

Рис. 3. Сравнение генеральной и выборочной функций распределения.

уровень значимости (если в результате эксперимента окажется, что $D_{N} \geq D_{N}(\alpha)$, то согласно критерию Колмогорова-Смирнова с уровнем значимости $\alpha$, оснований для принятия гипотезы не имеется). Для небольших выборок процентные значения $D_{N}(\alpha)$ легко найти по таблице, например [13]. В случае, когда $N \geq 100$, удобно использовать приближенную формулу

$$
\begin{aligned}
& D_{N}(\alpha) \approx \sqrt{\frac{1}{2 N}\left(y-\frac{1}{18 N}\left[\left(2 y^{2}-4 y-1\right)-\alpha^{3}\left(3 y^{2}-y+\frac{1}{2}\right)\right]\right)} \\
&-\frac{1}{6 N}, \\
& y=2 x_{\beta}^{2} \text { или } y \approx-\ln \frac{\alpha}{2},
\end{aligned}
$$

где $x_{\beta}-$ корень уравнения

$$
K\left(x_{\beta}\right)=\beta=1-\alpha, \quad K(x)=\sum_{k=-\infty}^{+\infty}(-1)^{k} e^{-2 k^{2} x^{2}},
$$

где $K(x)$ - функция распределения Колмогорова.

Итак, если наблюдаемое значение статистики критерия $D_{N}$ меньше вычисленного по формуле (9) (или найденного по таблице), то гипотезу о соответствии выборки генеральной совокупности с функцией распределения нет оснований отвергать. Результаты для различных объемов выборки приведены в табл. 4. Видно, что во всех случаях гипотеза о соответствии моделируемых случайных величин заданным законам распределения может быть принята с доверительной вероятностью 99\%.

Таблица 4. Значения $D_{N}$ для $\widetilde{v}_{\text {ext }}$ и $\widetilde{p}_{\text {ext }} ; D_{N}(\alpha)$ для разных выборок

\begin{tabular}{c|c|c|c|c|c|c|c}
\hline \multicolumn{2}{c|}{$N$} & $10^{6}$ & $10^{5}$ & $10^{4}$ & $10^{3}$ & $10^{2}$ & 10 \\
\hline \multirow{2}{*}{$D_{N}$} & $\widetilde{v}_{\text {ext }}$ & $5.73 \cdot 10^{-4}$ & $3.63 \cdot 10^{-3}$ & $9.02 \cdot 10^{-3}$ & $2.14 \cdot 10^{-2}$ & $4.60 \cdot 10^{-2}$ & $4.77 \cdot 10^{-1}$ \\
& $\widetilde{p}_{\text {ext }}$ & $5.48 \cdot 10^{-4}$ & $2.66 \cdot 10^{-3}$ & $7.27 \cdot 10^{-3}$ & $2.20 \cdot 10^{-2}$ & $8.43 \cdot 10^{-2}$ & $3.41 \cdot 10^{-1}$ \\
\hline \multirow{2}{*}{$D_{N}(\alpha)$} & 0.01 & $2.23 \cdot 10^{-3}$ & $7.04 \cdot 10^{-3}$ & $2.23 \cdot 10^{-2}$ & $7.02 \cdot 10^{-2}$ & $2.20 \cdot 10^{-1}$ & $6.56 \cdot 10^{-1}$
\end{tabular}




\section{Заключение}

В настоящей работе вылет электрона с поверхности катода рассмотрен как случайный процесс. Построены функции распределения электронов по полным энергиям и энергиям, связанным с нормальной компонентой импульса. Сформулировано и доказано утверждение о связи специального вида случайных значений энергий $\mathscr{E}^{*}$ и $\mathscr{E}_{z}^{*}$, с которыми электрон покидает эмиттер. Проведен розыгрыш этих энергий как зависимых случайных величин. Дополнительно осуществлена статистическая проверка гипотезы о соответствии выборок $\mathscr{E}^{*}$ и $\mathscr{E}_{z}^{*}$ генеральным совокупностям. С вероятностью 99\% гипотеза была принята на основе критерия Колмогорова-Смирнова. Были также проанализированы семейства квадратурных формул и способы прогнозирования погрешности для достижения заданной точности при интегрировании законов распределения. Для вычисления интегралов с переменным верхним пределом были построены интегральные интерполянты. В качестве оптимального подхода был выбран способ, основанный на комбинации методов Симпсона (с простым и удвоенным шагом) и Боде.

Исследование А.Ю. Антонова выполнено за счет гранта Российского научного фонда (проект № 15-19-30022), СПбГЭТУ „ЛЭТИ“. Н.В. Егоров и Н.С. Демченко осуществили вклад в работу на добровольной основе.

\section{Список литературы}

[1] Егоров Н.В., Шешин Е.П. Автоэлектронная эмиссия. Принципы и приборы. Долгопрудный: Изд. Дом Интеллект, 2011. $704 \mathrm{c}$.

[2] Виноградова Е.М., Егоров Н.В., Телевный Д.С. // ЖТФ. 2014. Т. 84. Вып. 2. С. 139-144.

[3] Nikiforov K., Trofimov V., Egorov N. // „Stability and Control Processes" in Memory of V.I. Zubov International Conference (SCP 2015). St. Petersburg, 2015. P. 180-182.

[4] Fowler R.H., Nordheim L.W. // Proc. Roy. Soc. A. 1928. Vol. 119. N 781. P. 173-181.

[5] Young R.D. // Phys. Rev. 1959. Vol. 113. N 1. P. 110-114.

[6] Антонов А.Ю., Демченко Н.С. // Вестник СПбГУ. 2010. Сер. 10. Вып. 3. С. 3-12.

[7] Nikiforov K.A., Andrievskiy D.S. // Proc. of the $20^{\text {th }}$ International Workshop on Beam Dynamics and Optimization (BDO 2014). 2014. P. 6890057.

[8] Егоров Н.В., Антонова Л.И., Антонов А.Ю. // Поверхность. Рентгеновские, синхротронные и нейтронные исследования. 2012. № 11. С. 41-46.

[9] Соболь И.М. Численные методы Монте-Карло. М.: Наука, 1973. $312 \mathrm{c}$.

[10] Hairer E., Norsett S.P., Wanner G. Solving ordinary differential equations I. Nonstiff problems. $2^{\text {nd }}$ ed. Springer, 2008. 528 p.

[11] Press W.H., Teukolsky S.A., Vetterling W.T., Flannery B.P. Numerical recipes. The art of scientific computing. $3^{\text {rd }}$ ed. Cambridge University Press, 2007. 1256 p.

[12] Matsumoto M., Nishimura T. // ACM T. Model. Comp. S. 1998. Vol. 8. N 1. P. 3-30.

[13] Большев Л.Н., Смирнов Н.В. Таблицы математической статистики. М.: Наука, 1983. 416 с. 